\title{
Identification of genomic regions associated with agronomical traits of bread wheat under two levels of salinity using GWAS
}

Fahad Alotaibi

King Abdulaziz City for Science And Technology

Rahmah N. Al-Qthanin

King Khalid University College of Science

Maha Aljabri

Umm Al-Qura University College of Applied Sciences

Mohammed Albaqami

Umm Al-Qura University College of Applied Sciences

Salah Abou-Elwafa ( $\sim$ elwafa75@aun.edu.eg )

Assiut University Faculty of Agriculture https://orcid.org/0000-0002-9018-598X

\section{Research Article}

Keywords: Triticum, Salt stress, Salinity, Tolerance index, Association analysis, QTL

Posted Date: August 23rd, 2021

DOI: https://doi.org/10.21203/rs.3.rs-837259/v1

License: @ (i) This work is licensed under a Creative Commons Attribution 4.0 International License. Read Full License 


\section{Abstract}

Soil salinity is a major environmental stress that adversely impacts the growth, development, productivity and quality of crop species, in particular, in arid and semi-arid regions. Identification of chromosomal regions associated with agronomic traits under salinity stress is crucial for improving salinity tolerance in wheat. GWAS and structure analyses were employed to evaluate 289 elite lines of the Wheat Association Mapping Initiative (WAMI) population under low (LS) and high (HS) salinity conditions using 15,737 SNP markers and seven agronomical traits.

Evaluated genotypes responded differently to the different environments in all measured phenotypic traits, highlighting genetic diversity within the WAMI population in response to salt stress. Heritability degree ranged from moderate (37\%) to high (88\%). GWAS identified 118 and 120 significant associations between SNP markers and seven evaluated phenotypic traits under LS and HS conditions, respectively. Significant association of some markers with more than one phenotypic trait was observed, indicating possible pleiotropic or indirect effects. A high degree of significant linkage disequilibrium (> 52\%) was observed among SNP markers on different chromosomes indicating epistatic interaction. The salt stress index (STI) exhibited a positive significant correlation to grain yield per plant (GYP) under both LS and HS conditions $\left(\mathrm{R}^{2}=0.851-0.856\right)$. A linear regression between STI and GYP under HS conditions (GYPS) was identified, suggest that STI is the best tolerance index for predicting high yielding-genotypes. The results present the WAMI population as a valuable source for improving yield potential for salt tolerance in wheat. Furthermore, our findings emphasize that GWAS is a powerful tool in promoting wheat breeding through accurate identification of molecular markers significantly associated with agronomic traits, which is essential for marker-assisted breeding.

\section{Introduction}

Soil salinity is a major environmental stress that negatively affects the growth, development, productivity and quality of crop species, in arid and semi-arid regions in particular. It inhibits the metabolic processes in the plants and thereby reduce the photosynthetic capacity and biomass accumulation (Che-Othman et al. 2020). An estimated 800 million hectares of irrigated soils worldwide are estimated to be salt affected (Hernández 2019). The extent and severity of salinity-affected soils is expected to be increased as a result of deficit drainage of irrigated soil, rising water tables and climate change (Aljabri et al. 2021; Alotaibi et al. 2021; Oyiga et al. 2018). Under salt stress conditions, plants exhibit considerable reduction in growth rates, increasing leaf senescence and reduced tillering, and during the long-term exposure to salinity the reproductive development is adversely affected, resulting in a significant reduction in grain yield (Munns and Tester 2008). Therefore, understanding the mechanisms of salinity tolerance in crop plants is essential for the development of better salinity response varieties with an improved tolerance to salt stress. These salt-stress tolerant varieties would ameliorate the productivity of crop plants under the anticipated scenario of increasing salinity adverse impacts to ensure sustainable food security for the growing population and avoid social perturbations resulting from starvation or food shortage (Abou-Elwafa and Shehzad 2020; Tanaka et al. 2015).

Wheat (Triticum aestivum L.), one of the earliest domesticated crops, is the major source of food and feed worldwide. The increasing demand in wheat consumption due to the rapid growth in the world population, necessitates sustainable wheat production to ensure global food security (Garcia et al. 2019). Wheat is adapted to a wide spectrum of climatic regions including those subjected to salinity stress (Abou-Elwafa and Shehzad 2020; Monneveux et al. 2012). A representative core collection of wheat genotypes is essential for ensuring a high degree of genetic diversity that is crucial selection process in wheat breeding programs (Matus and Hayes 2002).

At the molecular level, plants have evolved to survive and grow under salt stress (Lv et al. 2020). Plants exhibit considerable plasticity at the morphology, physiology, biochemistry and molecular levels to survive and resist or tolerate salt stress, including the expression of $\mathrm{Na}^{+}$and $\mathrm{K}^{+}$transporter genes. Rapid and efficient responses of plants to salt stress are essential for the survival, reproduction and potential yields of cereal crops (Lohani et al. 2019). Plants would evolve an adaptive salt-stress tolerance mechanism to differentiate across growth stages as a result of long-term exposure to salinity (Oyiga et al. 2018). Dissecting the genetic factors underlying slat-stress would facilitate breeding for improved abiotic stress tolerant cultivars which is the most feasible and costeffective strategy to fight against such a major abiotic stress (Abou-Elwafa 2016a; Abou-Elwafa and Shehzad 2018).

Genetic variations of salt-stress tolerance in bread wheat are limited and are mainly based on the ability of the plant to exclude $\mathrm{Na}^{+}$from leaf blades and maintaining an appropriate $\mathrm{K}^{+} / \mathrm{Na}^{+}$ratio (Oyiga et al. 2018). The limited application of marker-assisted breeding in improving crop plants to salt-stress tolerance might be due to the complexity of salinity stress with respect to polygenic inheritance pattern, low heritability and high degree of genotype-byenvironment interaction. However, recent advances in genetic and genomic strategies would facilitate unrevealing the genetic control of important agronomic traits under abiotic stress conditions in different crop plants (Abou-Elwafa 2016b; Nezhadahmadi et al. 2013). The shared genomic regions and crosscompatibility between wild species and cultivated relatives provide wild species as a promising donor of valuable alleles to enhance the gene pool required for crop breeding and improvement (Abou-Elwafa 2016b). Several studies employed genetic variations in agronomic traits evaluated under salt-stressed conditions to identify QTLs associated with salt tolerance at different growth stages in wheat (Ahmad et al. 2013; Munns et al. 1999; Rahnama et al. 2011; Schachtman et al. 1992). However, the main drawback of these studies is the reliance on bi-parental mapping populations that are characterized by poor resolution in detecting QTLs and considerable time and costs needed to develop the appropriate mapping population, in addition to the limited number of alleles that can be studied simultaneously at any given locus (Mahmoud et al. 2018).

Genome-wide association study (GWAS) has emerged to overcome the drawbacks of conventional QTL analysis and improve and accelerate breeding programs of crop plants. GWAS is a powerful approach that is used to enhance recent advances in genomic tools and statistical methods through efficient utilization of cumulative historical recombination and mutation events in a population to identify significant marker-trait associations (QTLs) with fine mapping resolution and less research effort. which may allow (Abou-Elwafa et al. 2019; Fleury et al. 2010). GWAS has proven as a useful approach for uncovering the complex genetic mechanisms governing tolerance to abiotic stress in several crop plants (Long et al. 2013; Turki et al. 2015). However, although numerous research reports on GWAS for salt tolerance have been published in crop species, little research implementing GWAS for the identification of salt-stress tolerance loci have been carried out in wheat (Hu et al. 2021; Li et al. 2020; Quan et al. 2021; Yu et al. 2020). GWAS has identified 42 QTLs 
significantly associated with 10 salt tolerance associated traits, from which 9, 16 and 17 QTLs are associated with physiological, shoot ionic and biomass traits, respectively (Chaurasia et al. 2020). Using 395675 SNP markers in a diversity panel of 323 wheat accessions, (Li et al. 2020) identified 269 significant associations between phenotypic traits and SNP markers, from which 22 are overlapping with QTLs identified by bi-parental QTL mapping. Furthermore, GWAS in a panel of 191 wheat accessions genotyped by Wheat660K SNP array identified 389 significant SNPs represent 11 QTLs associated with several phenotypic traits including plant height, spike length, thousand kernels weight and yield under different salt treatments, with an $\mathrm{R}^{2}$ ranging from 9.14 to $50.45 \%$. Repetitive and pleiotropic loci on different chromosomes were significantly identified to be linked to yield and yield-related traits such as thousand kernels weight, spike number and spike length under low salinity conditions (Hu et al. 2021). Another GWAS in a wheat panel comprises 317 accessions genotyped with the wheat $90 \mathrm{~K}$ SNP revealed 37 SNPs located at 16 unique loci each explaining $6.3-18.6 \%$ of the phenotypic variations. Ten loci are novel, whereas the remaining 6 are overlapped with previously reported genes or QTLs. Besides, 9 loci are detected for two or more traits, indicating the complexity of the genetic architecture of salt tolerance in wheat (Quan et al. 2021). Employing both marker-based and pedigree-based kinship analyses in a panel of 307 wheat accessions including local landraces, exotic cultivars used in Chinese breeding programs and Chinese cultivars subjected to a GWAS revealed that favorable haplotypes were introduced in some exotic cultivars as well as a limited number of Chinese landraces (Yu et al. 2020).

The current study was carried out to evaluate a structured wheat population consists of 289 elite spring bread wheat lines for salt stress tolerance. The genetic diversity in salt stress tolerance within the population was further estimated. Employing GWAS approach using 15,737 SNP markers to identify significant associations between SNP markers and 7 physiological and agronomical traits under two levels of salinity.

\section{Material And Methods}

\section{Plant material and field experiments}

A genetically diverse structured population consists of 289 elite spring bread wheat lines of the Wheat Association Mapping Initiative (WAMI) collected from several CIMMYT's wheat international nurseries worldwide (Abou-Elwafa and Shehzad 2020); Suppl. Table 1) was used. Experiments were performed in $2017 / 2018$ and 2018/2019 growing seasons at Sohag governate, Egypt (lat $26^{\circ} 56^{\prime} \mathrm{N}$, long $31^{\circ} 70^{\prime}$ and alt $61 \mathrm{~m}$ asl) at two experimental sites of low (control) and high salinity soils. Composite soil samples were collected from the upper $30 \mathrm{~cm}$ layer of each experimental site before the beginning of each growing season. The physical and chemical properties of the soil at the two experimental sites are shown in Suppl. Table 2. Seed were sown in the field with row space $20 \mathrm{~cm}$ and plant space $10 \mathrm{~cm}$ on November 25, 2017, and November 15, 2018, and harvested on April 13, 2018 and April 9, 2019. Irrigation was performed by drip irrigation with $20 \mathrm{~cm}$ distance between drippers. Each treatment was irrigated twice a week using a total amount of $440 \mathrm{~mm}$ during the growing season. The chemical analysis of irrigation water is shown in Suppl. Table 3.

\section{Phenotyping}

The number of days to heading (HD) was estimated as the number of days from planting to the date when $50 \%$ of the plants have headed. The portable chlorophyll meter (SPAD502, Japan) was used to measure the leaf chlorophyll content (LCC) at the flowering stage. At harvest, five yield and yield-related traits were measured as average values of 10 individual plants from each plot. The measured traits include: i) plant height (PH), the main stem height at maturity, ii) Spike number/ plant (SN), iii) spike length (SL) excluding awns, iv) thousand kernel weight (TKW) and v) Grain yield per plant (GYP). Salt-stress tolerance indices (DTIs) for the measured traits were calculated according to the following formulas:

\section{See formulas in the supplementary files section.}

\section{Genome-wide association and structure analyses}

The $90 \mathrm{~K}$ Illumina Infinitum SNP array and SNP processing (https://data.cimmyt.org/file.xhtml?fileld=3822\&datasetVersionld=332; (Sukumaran et al. 2018) were employed to generate SNP markers by genotyping the WAMI population. A total of 15,737 SNP markers were implemented to perform genomewide association analysis with the seven measured phenotypic traits. The Mixed Linear Model (MLM) with Structure and Kinship in the TASSEL software (Bradbury et al. 2007) was employed to identify significant marker-trait associations between phenotypic traits and SNP markers. The STRUCTURE software (MCMC) was employed to perform structure analysis with a burning length of 100000 and MCMC cycles of 1000000 (Pritchard et al. 200). Each K was run 3 times from K2 to K10 and based on results, the K7 estimate was selected and used in association. The significance of the tested molecular marker main effect at $\mathrm{P} \leq 0.0001$ was considered as an indicator for the significance of the marker-trait association (Chaurasia et al. 2020). Phenotypic data under either the control or stressed conditions were used to perform genome wide association analysis.

\section{Estimation of linkage disequilibrium}

The software Tassel 5 Version 20160901 was employed to calculate linkage disequilibrium (LD) between SNP markers using the entire set of wheat lines. LD was estimated separately for all loci pairs on different chromosomes. Significance $P$ values of LD for SNP pairs were estimated by permutation (10,000 simulations).

\section{Experimental design and statistical analyses}

Experiments were carried out in a three-replicates split-plot design arranged in a randomized complete block design (RCBD). The main plots were assigned to salinity stress and the sub-plots were assigned to the wheat genotypes. Plants from each of the wheat genotypes were sown in two lines, each $2 \mathrm{~m}$ in length. Estimation of the additive and dominance effects was performed using the genetic model as essentially described in (Gambel 1962). Heritability in broad sense was estimated as a proportion of the genotypic variance to the phenotypic variance according to (Allard 1960). The Proc Mixed of the SAS package 
version 9.2 (SAS 2008) was employed to perform analysis of variance (ANOVA), correlation and linear regression among stress indices of measured phenotypic traits.

\section{Results}

\section{Phenotypic evaluation}

All measured agronomical traits were significantly affected across different environments. Salt-stress significantly reduced the number of days to heading (HD), leaf chlorophyll content (LCC), plant height (PH), number of spikes/ plant (SN), spike length (SL), thousand kernel weight (TKW) and grain yield per plant (GYP) (Fig. 1; Suppl. Tables 3\&4). All measured traits were significantly reduced in the first growing season under both salinity treatments. The analysis of variance (ANOVA) revealed highly significant differences $(P \leq 0.01)$ among evaluated genotypes in all measured traits. The interaction of genotype $x$ environment significantly affected all measured traits (Table 1), indicating genetic diversity among the evaluated genotypes in their response to salt stress. Heritability degree ranged between moderate ( $37 \%$ for TKW under LS conditions in the second season) to high ( $88 \%$ for LCC under HS conditions in the first season; Table 1).

Table 1

Analysis of variance (ANOVA) for traits under individual environment

\begin{tabular}{|c|c|c|c|c|c|c|c|c|c|c|c|c|c|c|c|c|c|}
\hline & \multicolumn{5}{|c|}{ Low salinity $2017 / 2018$} & \multicolumn{5}{|c|}{ High salinity $2017 / 2018$} & \multicolumn{5}{|c|}{ Low salinity $2018 / 2019$} & \multicolumn{2}{|c|}{ High salinity 2} \\
\hline & $\sigma_{G}^{2}$ & $\sigma_{\mathrm{e}}^{2}$ & Mean & SD & $H^{2}$ & $\sigma_{G}^{2}$ & $\sigma_{\mathrm{e}}^{2}$ & Mean & SD & $H^{2}$ & $\sigma_{G}^{2}$ & $\sigma_{\mathrm{e}}^{2}$ & Mean & SD & $H^{2}$ & $\sigma_{G}^{2}$ & $\sigma_{\mathrm{e}}^{2}$ \\
\hline HD & 22.18 & 34.16 & 106.45 & 0.29 & 0.58 & 162.61 & 40.13 & 93.02 & 6.69 & 0.80 & 61.15 & 17.88 & 88.70 & 4.44 & 0.83 & 27.85 & 26.24 \\
\hline LCC & 52.68 & 30.06 & 102.23 & 3.21 & 0.76 & 142.76 & 33.88 & 89.37 & 6.13 & 0.88 & 89.33 & 25.68 & 89.58 & 5.26 & 0.86 & 34.66 & $27.3 \epsilon$ \\
\hline $\mathrm{PH}$ & 103.18 & 25.95 & 98.00 & 6.12 & 0.83 & 122.90 & 27.62 & 85.71 & 5.57 & 0.81 & 117.51 & 33.48 & 90.45 & 6.08 & 0.81 & 41.46 & $28.4 \varepsilon$ \\
\hline SN & 9.32 & 6.17 & 14.31 & 2.78 & 0.74 & 7.01 & 3.52 & 10.26 & 1.91 & 0.82 & 4.20 & 8.79 & 8.74 & 2.50 & 0.56 & 1.37 & 2.74 \\
\hline SL & 2.08 & 1.11 & 12.54 & 1.08 & 0.82 & 1.20 & 0.85 & 10.26 & 0.92 & 0.77 & 1.13 & 0.50 & 10.08 & 0.73 & 0.82 & 1.14 & 0.54 \\
\hline GYP & 41.09 & 18.52 & 23.61 & 4.21 & 0.81 & 29.21 & 57.55 & 15.15 & 6.48 & 0.74 & 22.21 & 7.41 & 12.91 & 2.84 & 0.85 & 6.98 & 3.59 \\
\hline TKW & 0.35 & 1.09 & 4.17 & 0.21 & 0.48 & 0.22 & 0.71 & 3.51 & 0.64 & 0.45 & 0.20 & 0.42 & 3.54 & 0.55 & 0.50 & 0.03 & 0.76 \\
\hline
\end{tabular}

\section{Stress tolerance indices for selection to salt stress}

Salt stress tolerance indices, i.e., STI, TOL, SSI and YSI, and their correlations and regressions to grain yield per plant under high and low-salinity conditions (GYPp and GYPs, respectively) of all evaluated genotypes for all measured traits in both growing seasons were estimated. However, because of the high degree of similarity between the correlations and regressions among stress tolerance indices of all measured traits, only the correlations and regressions for grain yield per plant were presented and further studied (Table 2). Linear regression analysis a revealed strong positive relationship between stress tolerance index (STI) and single plant yield under high salinity stress (GYPs) with an a $\mathrm{R}^{2}$ value of 0.834 . The higher the single plant yield under salt-stressed conditions the greater the STI value and the higher the tolerance of a wheat genotype to salt stress was observed (Fig. 2). Stress tolerance (TOL) revealed a highly significant positive correlation between grain yield per plant under low salinity conditions (GYPp), whereas its correlation with grain yield per plant under high salinity conditions (GYPs) was negative (Table 2), suggesting that employing TOL as a selection index causes a reduction in grain yield per plant under saltstressed conditions. Stress tolerance index (STI) exhibited highly significant positive correlations with either GYPp or GYPs, indicating that STI could be efficiently employed as a selection index for GYPp and GYPs compared to TOL, SSI and YSI. Grain yield per plant under low salinity conditions (GYPp) was linearly correlated with GYPs (Fig. 2), indicating that the high yielding elite genotype under low salinity conditions may produce reasonable yield under saltstressed conditions. In addition, the general linear regression revealed strong positive regressions between grain yield per plant under salt-stressed conditions and STI over the two growing seasons with a coefficient of determination ( $\left.R^{2}\right)$ of 0.834 (Fig. 2).

Table 2

Pearson correlation coefficients $(R)$ between salt stress indices and average plant grain yield over the two growing seasons.

\begin{tabular}{|c|c|c|c|c|c|}
\hline & GYPp & PGYs & STI & TOL & SSI \\
\hline GYPs & $0.520^{*}$ & & & & \\
\hline STI & $0.851^{\star \star}$ & $0.856^{\star *}$ & & & \\
\hline TOL & $0.956^{\star \star}$ & $-0.244^{\mathrm{ns}}$ & 0.670 & & \\
\hline SSI & $0.433^{*}$ & $-0.514^{\star *}$ & -0.048 & 0.670 ** & \\
\hline YSI & $-0.433^{*}$ & $0.514^{\star *}$ & 0.048 & $-0.670^{\star *}$ & $-1.00^{\star \star *}$ \\
\hline
\end{tabular}

\section{Genome-wide association analysis and population structure}


Genome-wide association analysis using 15,737 SNP markers and seven physiological, morphological, yield and yield-related traits under low and high salinity conditions identified 118 and 120 significant marker-trait associations under low and high salinity conditions, respectively, with an $\mathrm{R}^{2}$ values ranged from 3.91 to 17.59 (Figs. 3\&4; Suppl. Table 5).

Eleven SNP markers mainly clustered to chromosomes 1A and 1B exhibited significant association with the number of days to heading under low salinity conditions with an $\mathrm{R}^{2}$ value ranged between 3.91 and $4.87 \%$. Meanwhile, under high salinity conditions, the number of days to heading exhibited significant associations with 28 SNP markers with an $\mathrm{R}^{2}$ value ranged from 4.13 to $5.40 \%$. Out of those, 23 SNP markers are clustered to chromosome $5 \mathrm{~A}$ (Figs. $3 \& 4$; Suppl. Table 6). GWAS revealed 10 significant associations between leaf chlorophyll content and the SNP markers under either low or high salinity conditions with an $R^{2}$ value ranged between 3.41 and $4.67 \%$. The 10 significant associations under either environmental conditions are mainly clustered to chromosomes $2 \mathrm{~B}$ and 7A. A total of 17 SNP markers clustered mainly to chromosomes $6 \mathrm{~B}$ were significantly associated with plant height under low salinity conditions with an $R^{2}$ ranged between 4.15 and $6.47 \%$. Meanwhile, under low salinity conditions 15 significant marker-trait associations clustered to chromosomes $2 B$ and $6 \mathrm{~B}$ were identified for plant height under salt-stressed conditions with an $\mathrm{R}^{2}$ ranged between 3.82 and $7.66 \%$ (Figs. 3\&4; Suppl. Table 5). The number of spikes per plant exhibited 12 and 19 significant associations with the SNP markers under low and high salinity conditions, respectively, with an $\mathrm{R}^{2}$ value ranged between 3.98 and $7.46 \%$ (Figs. 3\&4; Suppl. Table 6). Out of the 15 significant marker trait associations identified for SN under low salinity conditions, 11 markers are located to chromosome $6 \mathrm{~A}$ and 2 markers are located to chromosome 7D. Under salt-stressed conditions, two major clusters consist of 7 and 5 significantly associated markers were identified for $\mathrm{SN}$ on chromosomes $2 \mathrm{~A}$ and $4 \mathrm{~B}$, respectively. The analysis identified 31 significant marker-trait associations clustered to four genomic regions on chromosomes $2 A, 2 B, 3 D$ and $5 B$ with spike length under low salinity conditions with an $R^{2}$ value ranged between 4.72 and $15.17 \%$. Under salt-stressed conditions, 13 SNP markers allocated to chromosomes 2B, 3B and 7B were significantly associated with spike length with an $\mathrm{R}^{2}$ value ranged between 4.31 and $14.71 \%$ (Figs. 3\&4; Suppl. Table 6). Twenty SNP markers clustered to three genomic regions on chromosome $2 B$, i.e., 88, 95-97 and $161 \mathrm{cM}$, are significantly associated with grain yield per plant under low salinity conditions $\left(R^{2}=5.20\right.$ and $\left.13.63 \%\right)$. Meanwhile, under salt-stressed conditions, 18 SNP markers located to chromosomes $2 \mathrm{~A}$, $4 \mathrm{~A}$ and $4 \mathrm{~B}$ exhibited significant association with grain yield per plant with an $\mathrm{R}^{2}$ value ranged from 5.38 and $15.81 \%$; Suppl. Table 6). GWAS revealed 17 significant marker-trait associations with thousand kernel weight under either normal or saltstressed conditions with an $\mathrm{R}^{2}$ value between 3.91 and 13.25\% (Figs. 3\&4; Suppl. Table 6). Under normal conditions, the SNP markers significantly associated with TKW are mainly (14 SNP markers) clustered to chromosome 1B, whereas under salt-stressed conditions they are distributed to chromosomes 1B, 2A, 4B, $5 \mathrm{~A}, 6 \mathrm{~A}, 6 \mathrm{~B}$ and $7 \mathrm{~A}$, with a major cluster of markers on chromosome 6A (Figs. 3\&4; Suppl. Table 6).

Structure analysis of the population was performed employing 15,737 SNP markers using the software package STRUCTURE v. 2.2. Based on a burn-in period of 100,000 iterations and implementing the iteration number for the MCMC algorithm to 100,000, the 289 lines were divided into 3 subpopulations according to the relative genetic distances using structure. The $\mathrm{K}$ was set from 2 to 10 and performed 9 runs for $\mathrm{K}$ values, the population structure matrix ( $\mathrm{Q}$ ) was estimated by running structure for $K=7$, from which the highest likelihood was obtained (Fig. 5). The LD analysis revealed a low to medium level of linkage disequilibrium among the 15,737 SNP markers ( $r 2$ ranging between 0.0 and 0.52 ) in this study (Suppl. Figure 1).

\section{Discussion}

Genome-wide association study (GWAS), which depends on the structure of linkage disequilibrium of alleles at different loci, is a powerful strategy for accurate identification and fine mapping of genomic regions underlying quantitative traits (Abou-Elwafa and Shehzad 2018; Simko et al. 2006). This approach is more efficient when the purpose is to identify quantitative traits associated with a single marker (Abou-Elwafa 2016b; Doerge 2002). However, the choice of a population is a pivotal factor in determining the resolution of association analysis in plants. So that, the selected population should exhibit a high degree of diversity with more extensive historical recombination to allow the detection of more alleles (Abou-Elwafa 2016a; Shehzad et al. 2009).

In the current study, the TASSEL software that implements a fixed-effects linear model was employed to identify the association between SNP marker alleles and agronomic quantitative traits under two levels of soil salinity in the highly genetically diverse Wheat Association Mapping Initiative (WAMI) population that is consists of 289 elite breeding lines (Abou-Elwafa and Shehzad 2020). Each association between the SNP markers and phenotypic traits was performed individually. Structure analysis of the WAMI population revealed significant genetic variations either under low or high salinity conditions which are necessary for effective selection for genotypes with improved salinity tolerance. The continuous variations observed in the evaluated agronomic traits phenotypic under both low and high salinity conditions over the two growing seasons indicate polygenic inheritance of all evaluated traits. Besides, the agronomic traits overall evaluated genotypes respond differently to salinity conditions, emphasizing the adverse impacts of high salinity on wheat crop. The linear regression relationship observed between grain yield per plant under low salinity conditions (GYPp) and salt-stressed conditions (GYPs) indicates that indirect selection for salinity tolerance based on the high-yielding potential under low salinity conditions might be efficient (Talebi et al. 2009).

Salt stress tolerance indices were employed for the identification of high-yielding wheat elite lines under low and high salinity conditions. Four stress tolerance indices, i.e., STI, TOL, SSI and YSI, were employed for the identification of high-yielding wheat elite lines under either low and high salinity conditions. Noteworthy, STI is the only salt-stress tolerance index (STI) that revealed positive correlations with the average grain yield per plant under either low and high salinity conditions over the two growing seasons, indicating that STI is the best stress tolerance index that could be efficiently employed for prediction of highyielding wheat genotypes under salt-stressed conditions. The results further indicate that employing the other stress tolerance indices, i.e., SSI, TOL, and YSI, in prediction for high productivity may not be efficient under variable environmental conditions (Abou-Elwafa and Shehzad 2020). Besides, the linear regression relationship detected between STI and the average grain yield per plant under high salinity conditions over the two growing seasons emphasizes that STI might be employed as a powerful selection index for prediction and selection of high-yielding genotypes under salinity stress conditions. Interestingly, none of the other estimated stress indices, i.e., TOL, SSI or YSI, revealed such a linear regression relationship when plotted against grain yield per plant (Fig. 6), indicating that none of those indices could not be successfully implemented for prediction and selection of high-yielding genotypes under salt-stressed conditions. 
Genome-wide association analysis using 15,737 SNP markers identifies 238 significant associations between SNP markers and the seven evaluated traits. The 11 and 28 significant associations identified between the number of days to heading (HD) and SNP markers under low and high salinity conditions, respectively, were clustered to chromosomes 1A, 1B, 7B and 5A. A major heading date QTL (Qhd.4W-5A.1) co-localized with the two major vernalization genes Vrn-A1 and Vrn-A2 on chromosome 5A has been identified (Guedira et al. 2016). Besides, a major cluster of molecular markers located to chromosome 5A has been reported to be significantly associated with heading date (Abou-Elwafa and Shehzad 2020). Additionally, a major heading date QTL associated with the primary photoperiod gene $P p d-B 3$ on chromosome 7B has been identified and genetically mapped (Fowler et al. 2016). The significant associations between LCC and SNP markers were mainly clustered to chromosomes $2 B$ and 7A. Furthermore, a major genomic region associated with LCC under either low and high salinity conditions located to the telomeric region of chromosome 7A, emphasizing that the LCC is mainly controlled by genetic factors (Dai et al. 2016) which is consistent with the high degree of heritability estimated for this trait (75-88\%) under either low and high salinity across the two growing seasons (Table 1). Moreover, a major QTL associated with chlorophyll b designated qChla7A was identified on chromosome 7A (Zhang et al. 2009). SNP markers significantly associated with $\mathrm{PH}$ were located to specific genomic regions on chromosomes $2 \mathrm{~B}, 3 \mathrm{~A}, 4 \mathrm{~B}, 5 \mathrm{~A}$ and $6 \mathrm{~B}$. Four genomic regions located on chromosomes $2 \mathrm{~B}, 3 \mathrm{~A}$ and 6B were significantly associated with $\mathrm{PH}$ under either LS and HS conditions, indicating that plant height is mainly controlled by genetic effect. The high heritability degrees observed for plant height (77-83\%) under all environmental conditions (Table 1) support this finding. Several PH-associated QTLs have been identified on chromosome 3A (Ali et al. 2011; El-Feki et al. 2018; Rustgi et al. 2013), however, majors plant height QTLs were identified on chromosome 4B (Zhang et al. 2018). The SNP marker RAC875_c24550_1150 located to chromosome 4B and associated with PH under high salinity conditions (Suppl. Table 6) has been previously identified to be associated with plant height QTLs under different environmental conditions (Abou-Elwafa and Shehzad 2020; Zou et al. 2017). Besides, the identification of a cluster of markers on chromosome 4B that are significantly associated with $\mathrm{PH}$ is consistent with the identification of a cluster of the gibberellic acid-insensitive reduced height genes (Rht1) that control plant height on chromosome 4B (Cabral et al. 2018; El-Feki et al. 2018). Furthermore, the identification of a cluster of markers co-localized with the genomic region of the Vrn-A1 gene that has a reducing effect on plant height (Chu et al. 2008), suggesting that Vrn-A1 might be a promising candidate for this QTL. A major cluster of the markers significantly associated spike number/ plant (SN) resides chromosome $6 \mathrm{~A}$ which is consistent with the identification of a genomic in close vicinity to the major tillering promoter $\mathrm{Gli}-\mathrm{A} 2$ (a multigene protein family gliadin) on chromosome 6A (Li et al. 2002). Furthermore, a major spike number related QTL was mapped to chromosome 7B (Shah et al. 1999) at which three significant marker-trait associations were identified for SN in our study. SNP markers significantly associated with SL were mainly clustered to chromosomes 2A, 2B, 3B, 3D, 5B and 7B, which is consistent with the results of (El-Feki et al. 2018) where 10 spike length QTLs were detected, three of which were mapped to chromosomes 2B, 3D and 7B. A major SL-associated QTL designated (QEl.fCu-3D; (Chu et al. 2008)was mapped to the short arm of chromosome 3D where a major cluster of 13 markers were identified to be significantly associated with SL under low salinity conditions in this study. Moreover, our results are in agreement with the identification of three SL-associated QTLs, i.e., QSI.cau-2B.2, QSI.cau-7B.1 and QSI.cau-7B.2, which are stable across different environmental conditions, on chromosomes $2 \mathrm{~B}$ and 7B, respectively (Zhai et al. 2016). Additional verification findings for the importance of chromosomes $2 \mathrm{~B}$ and $7 \mathrm{~B}$ in the control of SL in wheat are the identification of two common loci associated with SL on chromosomes 2B and 7B, with the chromosome 7B locus was significant and stable across five environments (Li et al. 2019).

Markers revealed significant associations with grain yield per plant (GYP) were mainly clustered to the genomic region of 88-97 cM of chromosome $2 \mathrm{~B}$ under low salinity conditions, two of which, i.e., wsnp_Ex_c20786_29875033 and BS00003404_51, are associated with SL, whereas under salt-stressed conditions five of the SL significantly associated markers located to chromosomes $2 \mathrm{~A}$ are also significantly associated with SN. The significant association of markers with more than one phenotypic trait indicates possible pleiotropic or indirect effects of the QTLs harboring those markers. In a similar GWAS, 12 significant associations between SNP markers and grain yield were identified on, from which 2 are located to chromosomes 2A and 2B (Li et al. 2019). The SNP markers significantly associated with TKW under low and high salinity conditions were clustered mainly to chromosomes 1B, 2A and 6A. Two markers, i.e., RFL_Contig785_535 and RFL_Contig785_1700, are located to chromosome 1B and explaining 8.22-13.25\% of the phenotypic variance in this trait were identified under both the normal and the stressed environments. Significant associations between those two markers and TKW were previously identified in a GWAS using the same WAMI population (Sukumaran et al. 2018). A major QTL significantly associated with marker BS00023092_51 under the salt-stressed conditions that is closely linked to the marker BS00036878_51 on chromosome 6A identified previously to be associated with TKW-QTL (Zou et al. 2017). In addition, the genomic region of chromosome 6A (78-85 cM) that harbors 7 SNP markers (BS00023092_51, Tdurum_contig76709_195, Excalibur_c56264_188, BobWhite_c17086_197, IAAV1263, BobWhite_c1082_134 and Tdurum_contig47663_321) has been earlier identified to harbor a major QTL for TGW (Sukumaran et al. 2018).

In conclusion, GWAS employing the TASSEL software revealed the identification of 238significant associations among SNP markers and seven phenotypic traits. Besides, the results revealed the significant associations of some SNP markers with more than one phenotypic trait, indicating possible pleiotropic or indirect effects of the QTLs associated with those markers. Our study identified significant associations between SNP markers and phenotypic traits with a high degree of genetic diversity controlling different mechanisms for adaptation to high salinity conditions. The high degree of significant linkage disequilibrium (> 52\%) observed among SNP markers on different chromosomes indicates epistatic interaction. The results present the WAMI population as a valuable source for improving yield potential for salt tolerance in wheat. Furthermore, our findings emphasize that GWAS is a powerful tool in promoting wheat breeding through accurate identification of molecular markers significantly associated with agronomic traits, which is essential for marker-assisted breeding.

\section{Declarations}

\section{Funding}

Not applicable.

\section{Competing interests}


The authors declare that there is no conflict of interest.

\section{Availability of data and material}

All data are included within the manuscript and its supplementary material.

\section{Code availability}

Not applicable.

\section{Authors' contributions}

SFA conceived the study, analyzed the data, performed the experiment, collected the data and wrote the manuscript. FA, RA, MAJ and MAB helped in data analysis and presentation. All authors read and approved the final version of the manuscript.

\section{Ethics approval and consent to participate}

Not applicable.

\section{Consent to Participate}

Not applicable.

\section{Consent for publication}

Not applicable.

\section{Acknowledgments}

The authors gratefully acknowledge the International Maize and Wheat Improvement Center (CIMMYT) for making the plant materials and genotypic data available. The authors gratefully acknowledge the great help from Dr. Tariq Shehzad, Syngenta, 3054 E Cornwallis Rd, Durham, NC 27709, USA, in performing the analysis of GWAS.

\section{References}

1. Abou-Elwafa SF (2016a) Association mapping for drought tolerance in barley at the reproductive stage. Cr Biol 339:51-59

2. Abou-Elwafa SF (2016b) Association mapping for yield and yield-contributing traits in barley under drought conditions with genome-based SSR markers. Comptes rendus biologies 339:153-162

3. Abou-Elwafa SF, Amin A, Shehzad T (2019) Genetic mapping and transcriptional profiling of phytoremediation and heavy metals responsive genes in sorghum. Ecotoxicol Environ Saf 173:366-372

4. Abou-Elwafa SF, Shehzad T (2018) Genetic identification and expression profiling of drought responsive genes in sorghum. Environ Exp Bot 155:12-20

5. Abou-Elwafa SF, Shehzad T (2020) Genetic diversity, GWAS and prediction for drought and terminal heat stress tolerance in bread wheat (Triticum aestivum L.). Genetic Resources and Crop Evolution

6. Ahmad M, Shahzad A, Iqbal M, Asif M, Hirani AH (2013) Morphological and molecular genetic variation in wheat for salinity tolerance at germination and early seedling stage. Australian Journal of Crop Science 7

7. Ali ML, Baenziger PS, Ajlouni ZA, Campbell BT, Gill KS, Eskridge KM, Mujeeb-Kazi A, Dweikat I (2011) Mapping QTL for Agronomic Traits on Wheat Chromosome 3A and a Comparison of Recombinant Inbred Chromosome Line Populations. Crop Sci 51:553-566

8. Aljabri M, Alharbi S, Al-Qthanin RN, Ismaeil FM, Chen J, Abou-Elwafa SF (2021) Recycling of beet sugar byproducts and wastes enhances sugar beet productivity and salt redistribution in saline soils. Environmental Science and Pollution Research

9. Allard RW (1960) Principles of Plant Breeding. John Wiley and Sons, New York

10. Alotaibi F, Bamagoos AA, Ismaeil FM, Zhang W, Abou-Elwafa SF (2021) Application of beet sugar byproducts improves sugar beet biofortification in saline soils and reduces sugar losses in beet sugar processing. Environmental Science and Pollution Research

11. Bouslama M, Schapaugh WT (1984) Stress Tolerance in Soybeans. I. Evaluation of Three Screening Techniques for Heat and Drought Tolerance1. Crop Sci 24:933-937

12. Bradbury PJ, Zhang Z, Kroon DE, Casstevens TM, Ramdoss Y, Buckler ES (2007) TASSEL: software for association mapping of complex traits in diverse samples. Bioinformatics 23:2633-2635

13. Chaurasia S, Singh AK, Songachan LS, Sharma AD, Bhardwaj R, Singh K (2020) Multi-locus genome-wide association studies reveal novel genomic regions associated with vegetative stage salt tolerance in bread wheat (Triticum aestivum L.). Genomics 112:4608-4621

14. Che-Othman MH, Jacoby RP, Millar AH, Taylor NL (2020) Wheat mitochondrial respiration shifts from the tricarboxylic acid cycle to the GABA shunt under salt stress. New Phytol 225:1166-1180

15. Chu C-G, Xu SS, Friesen TL, Faris JD (2008) Whole genome mapping in a wheat doubled haploid population using SSRs and TRAPs and the identification of QTL for agronomic traits. Mol Breeding 22:251-266 
16. Dai W, Girdthai T, Huang Z, Ketudat-Cairns M, Tang R, Wang S (2016) Genetic analysis for anthocyanin and chlorophyll contents in rapeseed. Ciência Rural 46:790-795

17. Doerge RW (2002) Mapping and analysis of quantitative trait loci in experimental populations. Nature reviews Genetics 3:43-52

18. El-Feki WM, Byrne PF, Reid SD, Haley SD (2018) Mapping Quantitative Trait Loci for Agronomic Traits in Winter Wheat under Different Soil Moisture Levels. Agronomy 8

19. Fernandez GCJ (1993) Effective selection criteria for assessing plant stress tolerance. In: 'Adaptation offood crops to temperature and water stress. AVRDC, Shanhua, Taiwan

20. Fischer R, Maurer R (1978) Drought resistance in spring wheat cultivars. I. Grain yield responses. Aust J Agric Res 29:897-912

21. Fleury D, Jefferies S, Kuchel H, Langridge P (2010) Genetic and genomic tools to improve drought tolerance in wheat. J Exp Bot 61:3211-3222

22. Fowler DB, N'Diaye A, Laudencia-Chingcuanco D, Pozniak CJ (2016) Quantitative Trait Loci Associated with Phenological Development, Low-Temperature Tolerance, Grain Quality, and Agronomic Characters in Wheat (Triticum aestivum L.). PLOS ONE 11:e0152185

23. Gambel EE (1962) Gene effects in corn (Zea mays L.) I.Separation and relative importance of gene effects for yield. Can J Plant Sci $42: 10$

24. Garcia M, Eckermann P, Haefele S, Satija S, Sznajder B, Timmins A, Baumann U, Wolters P, Mather DE, Fleury D (2019) Genome-wide association mapping of grain yield in a diverse collection of spring wheat (Triticum aestivum L.) evaluated in southern Australia. PLoS One 14:e0211730

25. Guedira M, Xiong M, Hao YF, Johnson J, Harrison S, Marshall D, Brown-Guedira G (2016) Heading Date QTL in Winter Wheat (Triticum aestivum L.) Coincide with Major Developmental Genes VERNALIZATION1 and PHOTOPERIOD1. PLOS ONE 11:e0154242

26. Hernández JA (2019) Salinity Tolerance in Plants: Trends and Perspectives. Int J Mol Sci 20:2408

27. Hossain ABS, Sears RG, Cox TS, Paulsen GM (1990) Desiccation Tolerance and Its Relationship to Assimilate Partitioning in Winter Wheat. Crop Sci 30:622-627

28. Hu P, Zheng Q, Luo Q, Teng W, Li H, Li B, Li Z (2021) Genome-wide association study of yield and related traits in common wheat under salt-stress conditions. BMC Plant Biol 21:27

29. Li F, Wen W, Liu J, Zhang Y, Cao S, He Z, Rasheed A, Jin H, Zhang C, Yan J, Zhang P, Wan Y, Xia X (2019) Genetic architecture of grain yield in bread wheat based on genome-wide association studies. BMC Plant Biol 19:168

30. Li L, Peng Z, Mao X, Wang J, Li C, Chang X, Jing R (2020) Genetic insights into natural variation underlying salt tolerance in wheat. J Exp Bot 72:11351150

31. Li WL, Nelson JC, Chu CY, Shi LH, Huang SH, Liu DJ (2002) Chromosomal locations and genetic relationships of tiller and spike characters in wheat. Euphytica 125:357-366

32. Lohani N, Golicz AA, Singh MB, Bhalla PL (2019) Genome-wide analysis of the Hsf gene family in Brassica oleracea and a comparative analysis of the Hsf gene family in B. oleracea, B. rapa and B. napus. Funct Integr Genomics 19:515-531

33. Long NV, Dolstra O, Malosetti M, Kilian B, Graner A, Visser RG, van der Linden CG (2013) Association mapping of salt tolerance in barley (Hordeum vulgare L.). Theor Appl Genet 126:2335-2351

34. Lv B, Wu Q, Wang A, Li Q, Dong Q, Yang J, Zhao H, Wang X, Chen H, Li C (2020) A WRKY transcription factor, FtWRKY46, from Tartary buckwheat improves salt tolerance in transgenic Arabidopsis thaliana. Plant physiology and biochemistry: PPB 147:43-53

35. Mahmoud AF, Abou-Elwafa SF, Shehzad T (2018) Identification of charcoal rot resistance QTLs in sorghum using association and in silico analyses. Journal of Applied Genetics

36. Matus IA, Hayes PM (2002) Genetic diversity in three groups of barley germplasm assessed by simple sequence repeats. Genome 45:1095-1106

37. Monneveux P, Jing R, Misra SC (2012) Phenotyping for drought adaptation in wheat using physiological traits. Front Physiol 3:429

38. Munns R, Hare RA, James RA, Rebetzke GJ (1999) Genetic variation for improving the salt tolerance of durum wheat. Aust J Agric Res 51:69-74

39. Munns R, Tester M (2008) Mechanisms of salinity tolerance. Annu Rev Plant Biol 59:651-681

40. Nezhadahmadi A, Prodhan ZH, Faruq G (2013) Drought tolerance in wheat. ScientificWorldJournal 2013:610721

41. Oyiga BC, Sharma RC, Baum M, Ogbonnaya FC, Léon J, Ballvora A (2018) Allelic variations and differential expressions detected at quantitative trait loci for salt stress tolerance in wheat. Plant Cell Environment 41:919-935

42. Quan X, Liu J, Zhang N, Xie C, Li H, Xia X, He W, Qin Y (2021) Genome-Wide Association Study Uncover the Genetic Architecture of Salt Tolerance-Related Traits in Common Wheat (Triticum aestivum L.). Front Genet 12:663941-663941

43. Rahnama A, Munns R, Poustini K, Watt M (2011) A screening method to identify genetic variation in root growth response to a salinity gradient. J Exp Bot 62:69-77

44. Rustgi S, Shafqat MN, Kumar N, Baenziger PS, Ali ML, Dweikat I, Campbell BT, Gill KS (2013) Genetic dissection of yield and its component traits using high-density composite map of wheat chromosome 3A: bridging gaps between QTLs and underlying genes. PloS one 8:e70526-e70526

45. SAS (2008) SAS/STAT® 9.2 user's guide. SAS Institute Inc., Cary

46. Schachtman DP, Lagudah ES, Munns R (1992) The expression of salt tolerance from Triticum tauschii in hexaploid wheat. Theor Appl Genet 84:714-719

47. Shehzad T, Iwata H, Okuno K (2009) Genome-wide association mapping of quantitative traits in sorghum (< i Sorghum bicolor (L.) Moench) by using multiple models. Breeding Science 59:217-227

48. Simko I, Haynes KG, Jones RW (2006) Assessment of linkage disequilibrium in potato genome with single nucleotide polymorphism markers. Genetics 173:2237-2245 
49. Sukumaran S, Reynolds MP, Sansaloni C (2018) Genome-Wide Association Analyses Identify QTL Hotspots for Yield and Component Traits in Durum Wheat Grown under Yield Potential, Drought, and Heat Stress Environments. Front Plant Sci 9:81

50. Talebi R, Fayaz F, Naji AM (2009) Effective selection criteria for assessing drought stress tolerance in durum wheat (Triticum durum Desf.). General Applied Plant Physiology 35:64-74

51. Tanaka A, Takahashi K, Masutomi Y, Hanasaki N, Hijioka Y, Shiogama H, Yamanaka Y (2015) Adaptation pathways of global wheat production: Importance of strategic adaptation to climate change. Sci Rep 5:14312

52. Turki N, Shehzad T, Harrabi M, Okuno K (2015) Detection of QTLs associated with salinity tolerance in durum wheat based on association analysis. Euphytica 201:29-41

53. Yu S, Wu J, Wang M, Shi W, Xia G, Jia J, Kang Z, Han D (2020) Haplotype variations in QTL for salt tolerance in Chinese wheat accessions identified by marker-based and pedigree-based kinship analyses. The Crop Journal 8:1011-1024

54. Zhai H, Feng Z, Li J, Liu X, Xiao S, Ni Z, Sun Q (2016) QTL Analysis of Spike Morphological Traits and Plant Height in Winter Wheat (Triticum aestivum L.) Using a High-Density SNP and SSR-Based Linkage Map. Frontiers in plant science 7:1617-1617

55. Zhang K, Zhang Y, Chen G, Tian J (2009) Genetic analysis of grain yield and leaf chlorophyll content in common wheat. Cereal Research Communications 37:499-511

56. Zou J, Semagn K, lqbal M, Chen H, Asif M, N'Diaye A, Navabi A, Perez-Lara E, Pozniak C, Yang R-C, Randhawa H, Spaner D (2017) QTLs associated with agronomic traits in the Attila $\times$ CDC Go spring wheat population evaluated under conventional management. PLOS ONE 12:e0171528

\section{Figures}
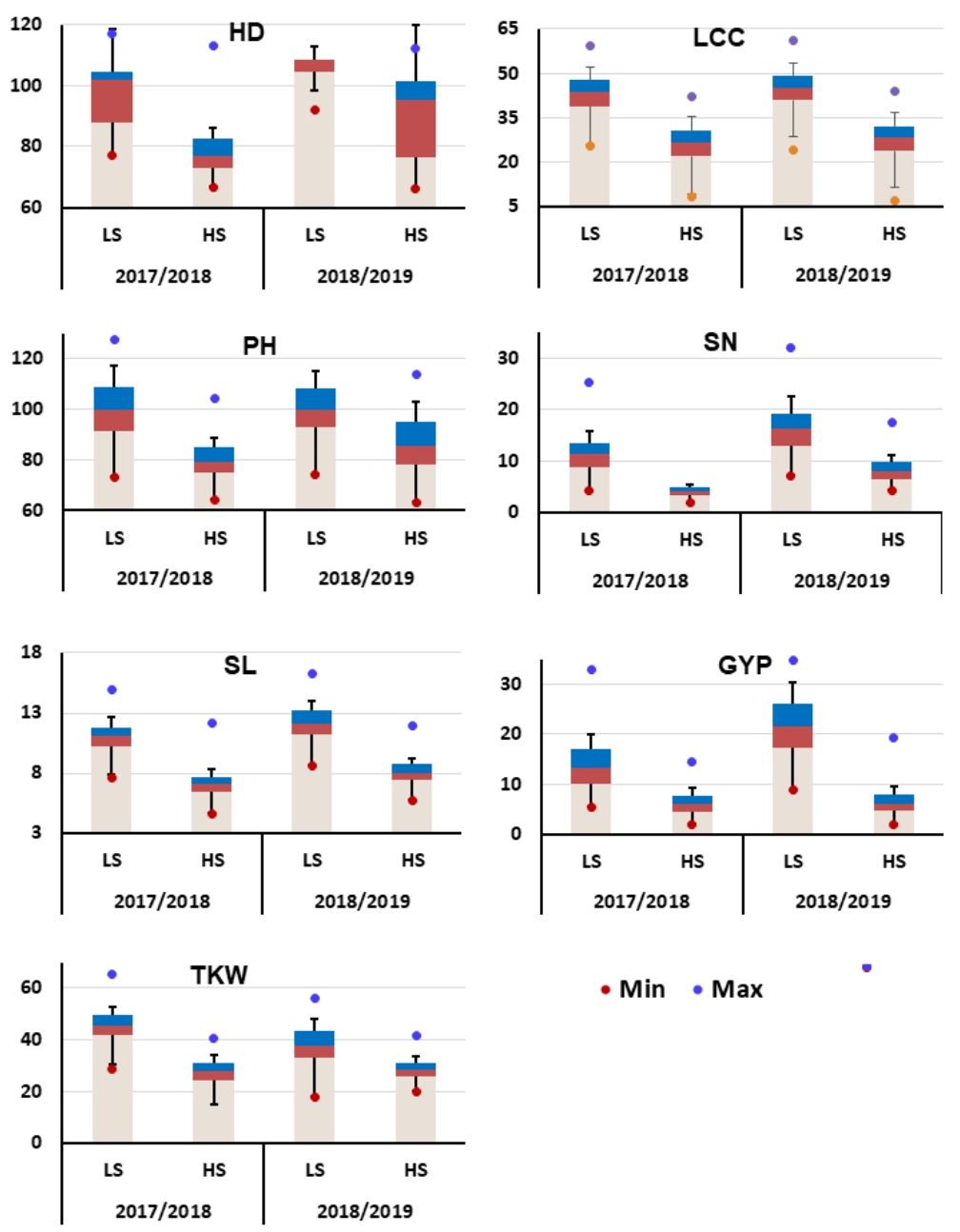

- Min - Max

\section{Figure 1}

Boxplot for seven agronomical traits of 289 genotypes of the WAMI population evaluated under low (LS) and high (HS) salinity conditions during the 2017/2018and 2018/2019 growing seasons. 

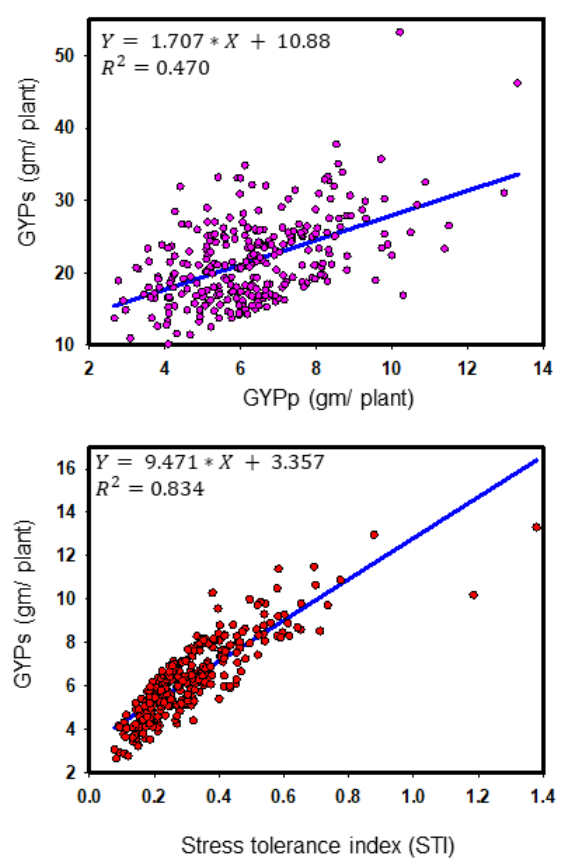

Figure 2

A) Relationship between grain yield per plant under low salinity (GYPp) and high salinity (GYPs) conditions over the two growing seasons. B) The relationship between grain yield per plant under high salinity conditions (GYPs) and salt-stress tolerance index (STI) over the two growing seasons. 

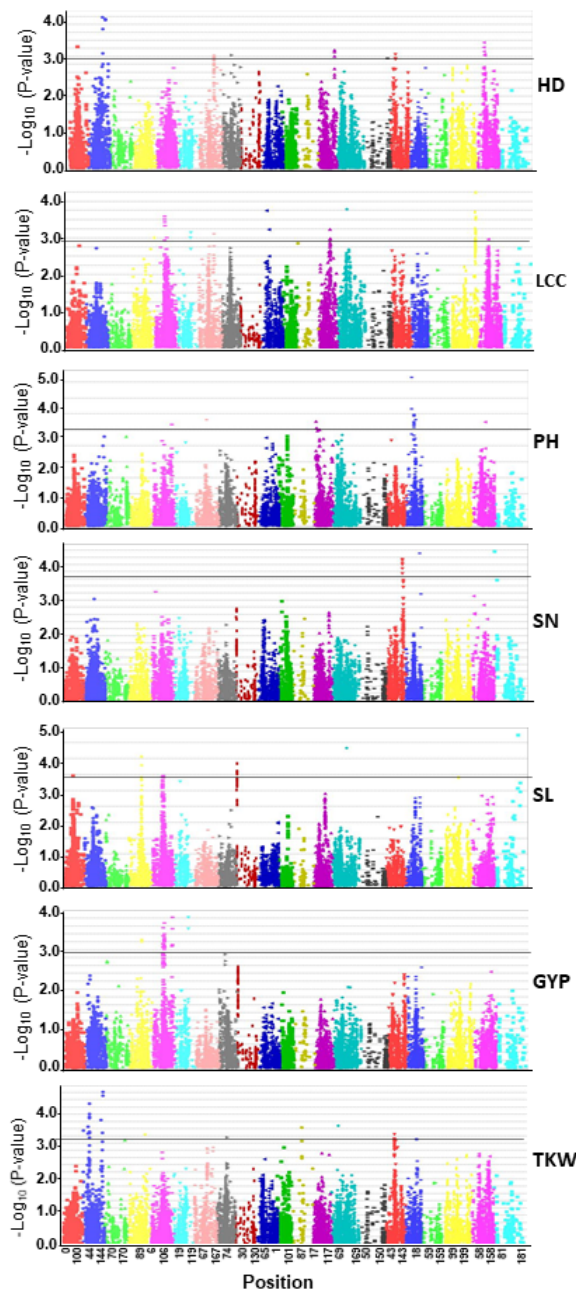

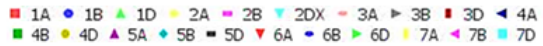

\section{Figure 3}

GWAS using 17,737 SNP markers and 289 WAMI for number of days to heading (HD), leaf chlorophyll content (LCC), plant height (PH), spike number (SN), spike length (SL), grain yield per plant (GYP) and thousand kernel weight (TKW) under low salinity conditions over the two growing seasons. 

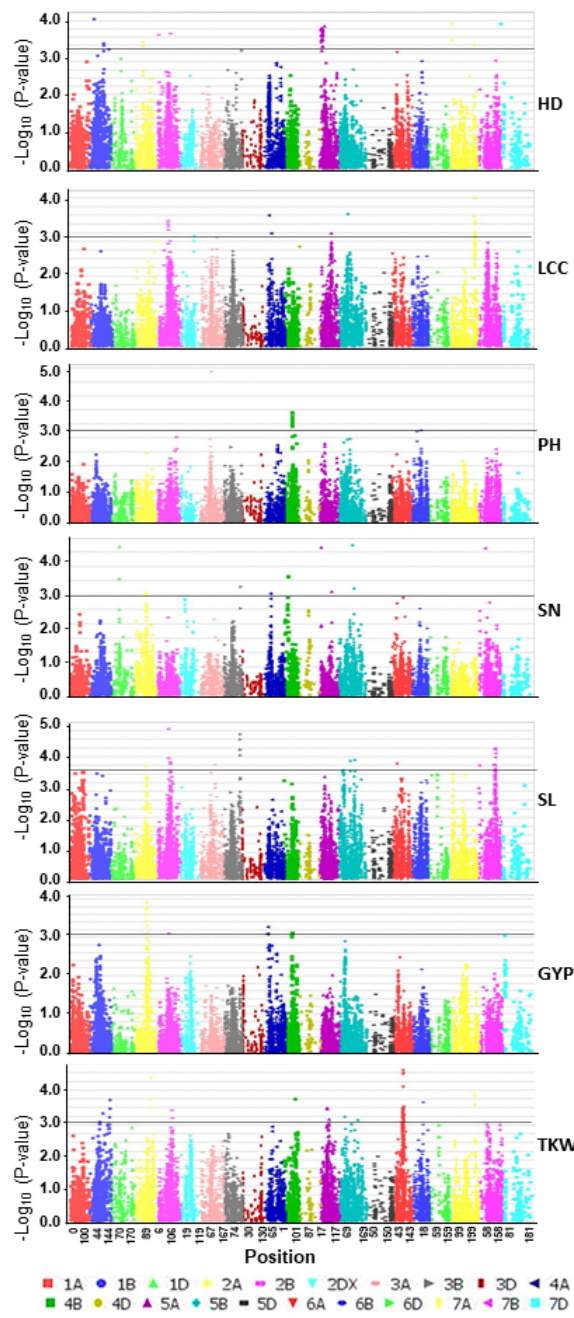

\section{Figure 4}

GWAS using 17,737 SNP markers and 289 WAMI for HD, LCC, PH, SN, SL, GYP and TKW under high salinity conditions over the two growing seasons. 

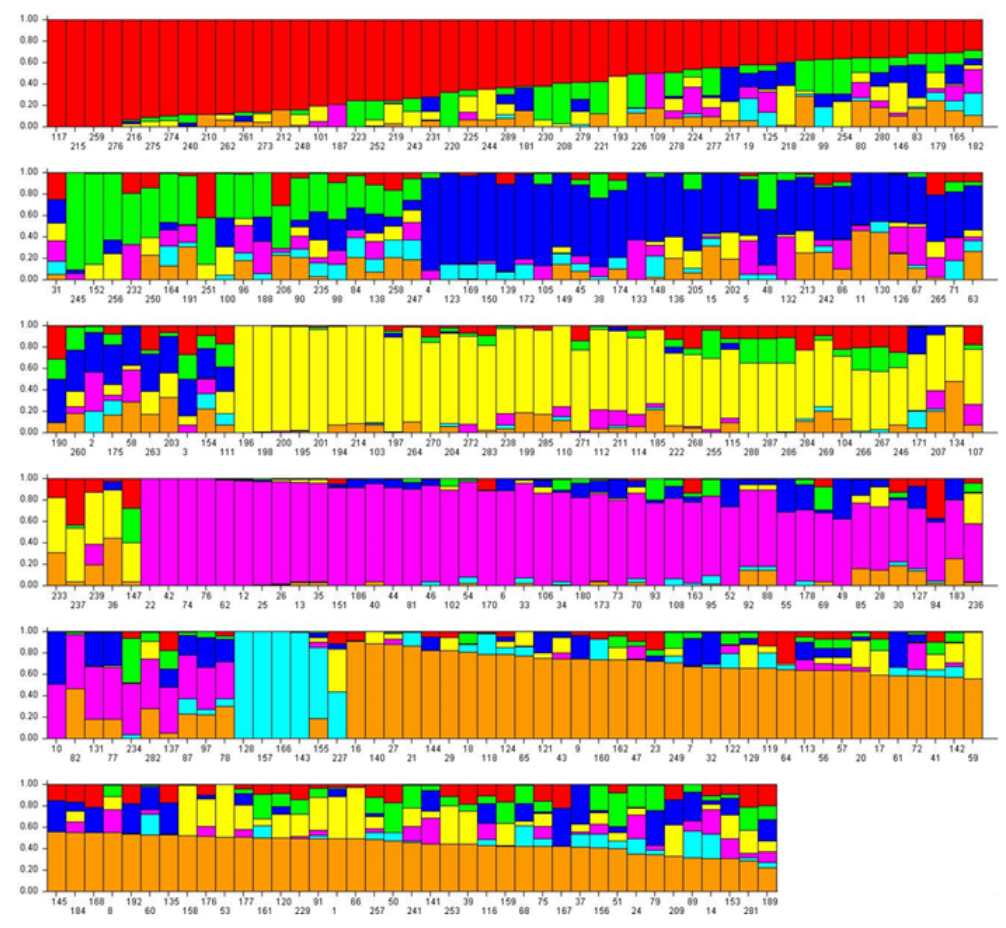

\section{Figure 5}

Distribution of 289 wheat lines within the 7 groups. Each genotype is correspondent to a single vertical colored bar above the diagram defragmented into $\mathrm{K}$ differently colored fragments. The length of the colored fragment is equivalent to each one of the $\mathrm{K}$ inferred subgroups. 

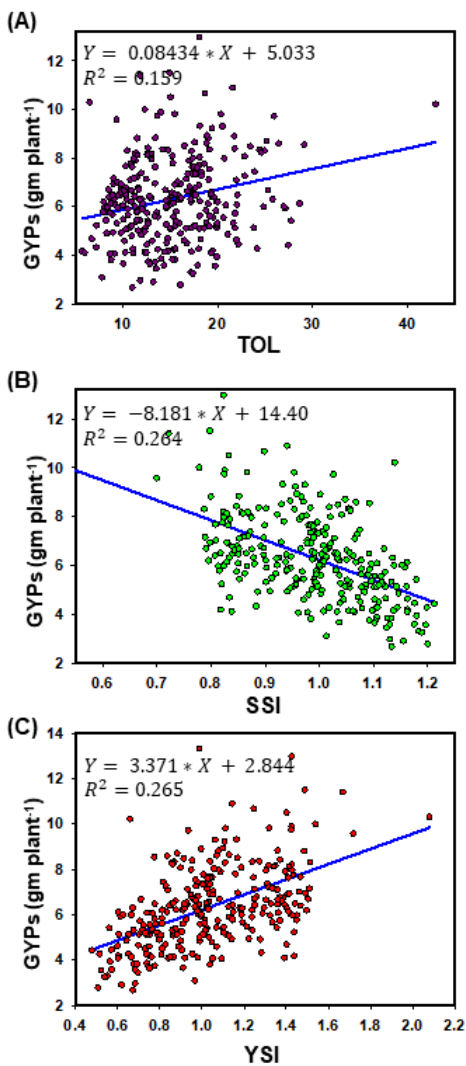

\section{Figure 6}

Relationship between grain yield per plant under high salinity conditions (GYPs) and salt tolerance indices (TOL, SSI and YSI) over the two growing seasons.

\section{Supplementary Files}

This is a list of supplementary files associated with this preprint. Click to download.

- formula.docx

- SupplTable1.docx

- SupplTable2.docx

- SupplTable3.docx

- SupplTable4.docx

- SupplTable5.docx

- Suppl.Fig.1.pptx

- Suppl.Table6.docx 